\title{
Reliability study of mechatronic power components using spectral photon emission microscopy
}

\author{
Niemat Moultif, Eric Joubert, Olivier Latry \\ Normandy University, University of Rouen, Groupe Physique des Matériaux, UMR CNRS 6634 \\ Site de Madrillet, Avenue de l'Université - B.P 12. St Etienne de Rouvray 76801 France \\ *corresponding author: eric.joubert@univ-rouen.fr
}

\begin{abstract}
In this paper, we present one of the most important failure analysis tools that permits the localizing and the identification of the failure mechanisms. It is a new spectral photon emission system, enabling to localize the failure and quickly get the photon emission spectra that characterize the failure with high resolution. A diffraction grating is used as a spectrometer in the system. Application results on mechatronic power devices such as HEMT AlGaN/GAN and SiC MOSFETs are reported.
\end{abstract}

\section{Introduction}

Failure analysis (FA) is becoming a crucial part of the integrated circuit (IC) design development and manufacturing. The microelectronic failure analysis process consists of five main steps, namely: failure validation, fault localization, sample preparation and defect tracing, defect characterization and root cause determination [1].

Fault localization is the step where the defective area is isolated to a smaller area. Photon emission microscopy (PEM) has been and still remains one of the most important localization techniques in the FA of semiconductor ICs. The major advantage of this technique is that it does not damage healthy devices nor further degrade existing defects. It is a non-destructive technique that requires the units to be in a fully functional state. But PEM technique has its limitation. It can only be used to indicate the place of the failure. In most cases it is not enough to allow an identification of the failure [2].

However, the study of spectral PE signatures gives a characterization of the defects, because each failure mechanism has its own unique spectral signature. Actually, the spectrum for a particular failure mechanism is distinguished from that of other mechanisms by its wavelength-dependent spectral shape and the wavelength range [3].

This paper introduces firstly the basics of PE phenomena, and a state of the art on the spectral photon emission systems (SPEMS). Next, the system developed is described. Then, the application results are presented on two technologies of semi-conductors devices.

\section{PE distribution characterization}

The PE technique is generally implanted on an optical microscope using a high sensible camera enabling the detection of the weak emissions from semiconductor devices. Fig. 1 shows the quantum efficiency of the best detector candidates used for observing photon emissions ( $\mathrm{Si}$ CCD, MCT and InGaAs).

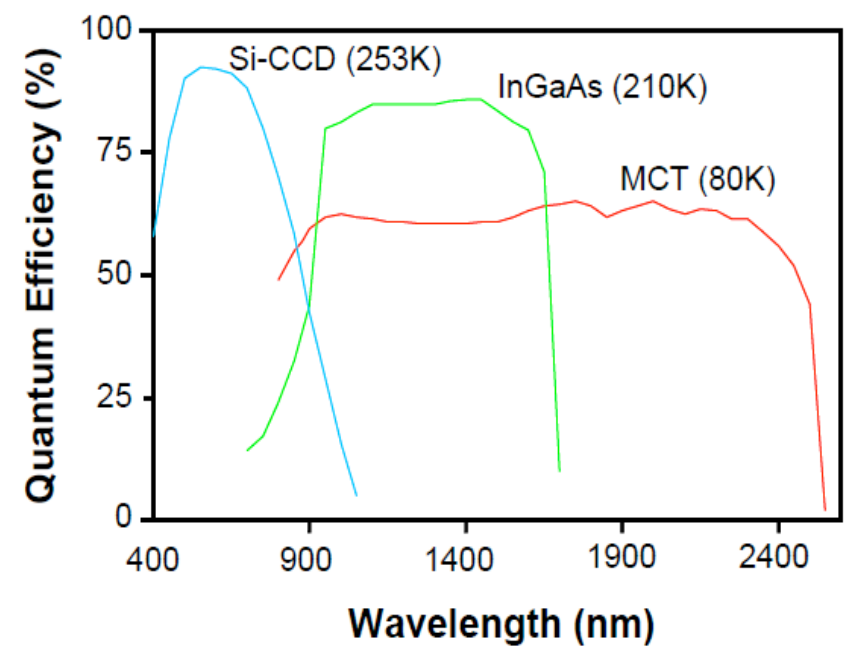

Fig. 1. Quantum efficiency of typical Si-CCD, MCT and InGaAs focal plane arrays [4]

In our system, PEM observations are performed using the microscope PHEMOS 1000 of HAMAMTSU. The system is equipped with a cooled Si-CCD detector with spectral sensitivity in the visible and near infrared range. The test procedure consists of three separate acquisitions: - Visible pattern image of the device under test (DUT), is obtained by illumination the DUT with an external light source Fig. (2-a).

- Noise image aim at measuring the interference. It is obtained when the DUT and the external light source are in the idle mode.

- Emission image, which is the result of the acquisition of the biased DUT emissions without illumination, under dark conditions (Fig. 2-b). 
The result of subtraction of the emission image by the noise image is superimposed with the visible image to localize the defective region of the DUT (Fig. 2-c).

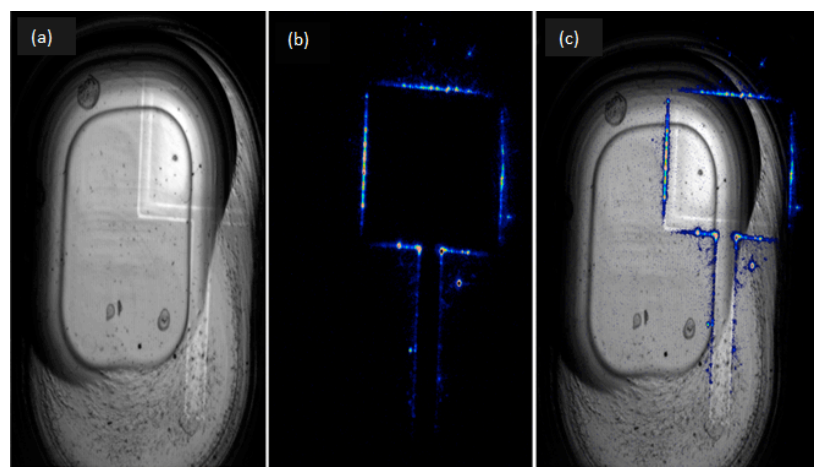

Fig. 2. (a) Pattern image of a backside opened Power MOSFET with the (x5) power lens. (b) Emission image. (c) Superimposed image.

The PE technique requires a detector view of the active surface. So, sample preparation and decapsulation of packaged devices are essential. Conventional frontside PE analysis is facing severe limitations with the growing use of multi-level metals and bridges, which prevent photon emission of the defects from reaching the detector. To overcome this problem we access the device from the backside, through the substrate. Since the transmission rate of light in the most conventional substrate $(\mathrm{Si}, \mathrm{SiC}$, and $\mathrm{GaN}$ ) is a function of wavelength, substrate thickness and the dopant concentration, backside analysis requires proper backside sample preparation and substrate thinning (less than $180 \mu \mathrm{m}$ thick) [5].

The PE process in a semiconductor occurs when there is a radiative emission of electromagnetic waves as a result of transition of carriers from a higher energy state to a lower energy state. There are different kinds of transition: Hot electron, hot hole, donor level, acceptor level, and deep level (Fig. 3).

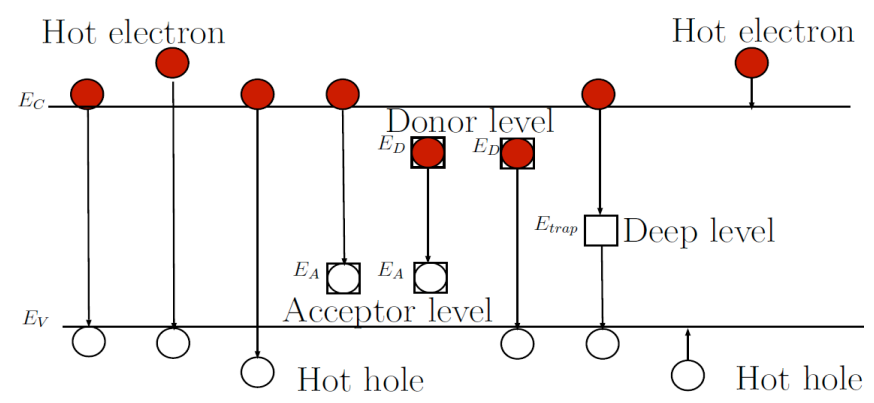

Fig. 3. Type of transitions

Since the wavelengths or energy of these emitted photons are related to the type of transitions of the carriers, this information can be a mean to determine the type of failure mechanisms that are emitting these photons. Whence comes the interest in extracting the spectral photon emission signature.

\section{PE Spectrum analysis}

\subsection{Failure analysis}

The light emission phenomena may be classified largely into three groups namely Oxide radiation, hot carrier radiation and recombination radiation. Each group is characterized by different spectral distributions [6] (Fig 4). In fact, each failure mechanism such as those caused by weak oxides, hot-carrier effects, leaky junctions, IC latchup, or other current leakage problems has a unique spectral signature.

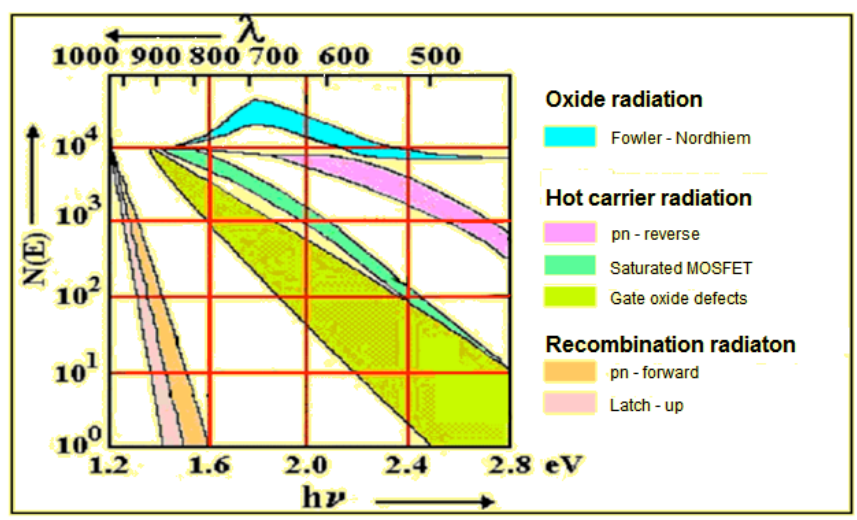

Fig. 4. Typical emission spectrum of most defects [6]

\subsection{Existing SPEM systems}

Using information obtained with a SPE system (SPES), it is possible to extract the fingerprint of the defect emissions of the DUT which can be correlated with the failure mechanism.

The oldest and the simplest approach for spectral analysis is the use of a serie of discrete bandpass filters of different wavelength range, to cover the whole spectrum in the detector spectral range response. By inserting different filters in the light path of the PEM, acquisition time becomes longer which may degrade further the device [6]. This represents a major disadvantage of this method. Moreover, these filters have low transmittance which makes the system less sensitive to weak emissions.

Other methods for spectroscopic analysis with the PEM include the use of an elliptical mirror [7, 8]. In the elliptical mirror method, Liu et al. used two separate detectors (one coupled to the spectrometer and one for the emission microscope) which make this method too expensive. In fact, the elliptical mirror is positioned between the sample and the microscope, to collect the light emitted from the sample and guided by an optical fiber to a monochromator. However, the system has a short working distance, making it difficult to work on encapsulated devices and unpackaged devices where probing are needed.

Another configuration is the in-lens method: a light dispersing element such as prism [9, 11, 12, 13] is positioned in the optical microscope to disperse the light emitted from the device to obtain a continuous wavelength 
spectrum. Many studies used the prism as spectrometer. De Kort et al. used a prism/lens system to focus the spectrum of the emitted light on the detector $[9,10]$. In this case, a beamsplitter is inserted between the objectives of the PEM and the detector. The reflected light is directed through a direct vision prism coupled by a focusing lens to a second detector.

A simplification of this method is presented by $\mathrm{M} \mathrm{S}$. Rasras et al. who used only one detector for both conventional failure analysis and continuous wavelength spectroscopy [11]. However, this system needs physical movement of the detector when switching between imaging and spectroscopic analysis.

An improved system based on a three element prism was realized by SL Tan et al. The three prisms are positioned in the path of the optical microscope, between the objective and the tube lens [12]. A more advanced system is proposed by $\mathrm{P}$. Scholz et al . who used only one prism placed in the optical path of the microscope [13]. The major disadvantage of the prism is its incapability to show the original emission position and the spectra at the same time. Moreover, this device is difficult to insert in the optical path due to the volume taken by it.

As a compromise between the prism and band pass filters, a diffraction grating was chosen for our system.

\subsection{System Description}

A diffraction grating was used as a spectrometer and placed in the optical path of the microscope, between the objective lenses and the Si-CCD detector (Fig. 5). The choice of a transmission grating was mainly motivated by its shape, high resolution and one shot continuous acquisition. In fact, the device is flat, easy to insert into the optical path and provides continuous registration between the origin of the emissions and the spectral distribution. Single emission images are treated to obtain the spectrum by expansion of the emission spot to a spectral tail

The system calibration is performed in two phases. The first phase aims to convert the raw PE spectrum as read out from the image into the real spectrum. This was obtained by founding the relation between the pixel number in the PEM image and the wavelength of the light. While the second phase has as purpose, the correction of the raw PE intensity spectrum by the known spectral response of the detector Si-CCD.

The grating chosen is a 100 lines/mm transmission diffraction grating, blazed in the first order.

The PE spectrum is extracted from the diffraction line. This line is drawn automatically by linking the centers of gravity of the points of orders ' 0 ' and ' 1 ' or based on average of the maximum intensity. The image processing is performed using personal software developed on Matlab.

\subsection{Spectral PEM experimental results}

\subsubsection{Reliability study of an AlGaN/GaN HEMT}

The first device studied in this work by the spectral PEM technique, is an $\mathrm{AlGaN} / \mathrm{GaN}$ high electron mobility transistor (HEMT) power bars on SiC substrate (European technology).

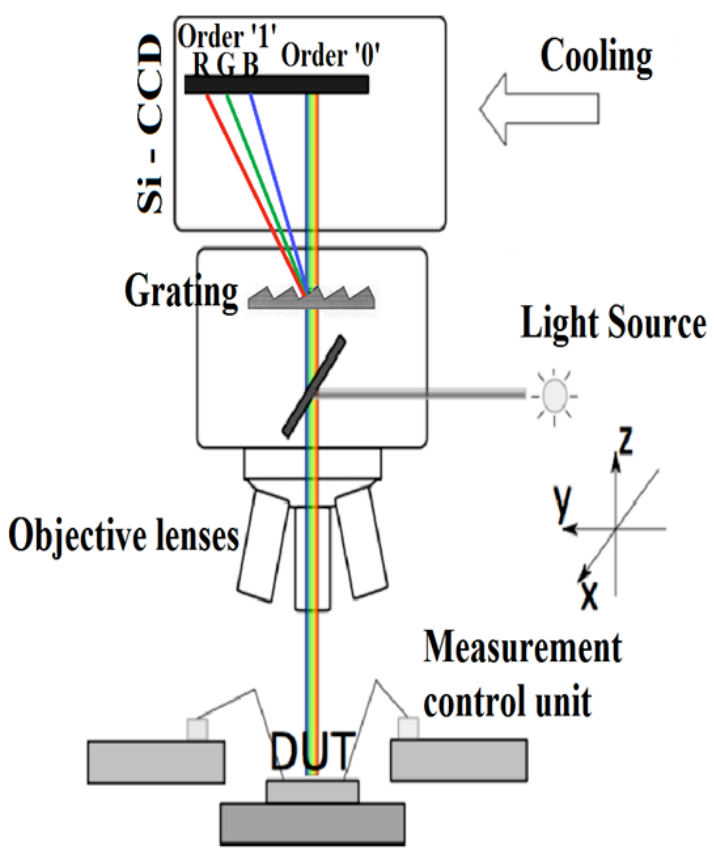

Fig. 5. Insertion of a grating in the optical path of the microscope.

PE Characterizations have been realized on the transistor mode $(\mathrm{Vgs}, \mathrm{Vds})=(-7 \mathrm{~V}, 30 \mathrm{~V})$. The current Ids is extremely low since the transistor is strongly pinched off.

The PE signatures are localized in this device along the gate fingers due to gate current flow. The PE inhomogeneity is visible in this pinched off mode when no significant current flows through the device. In fact, there are plenty of locations characterized by stronger PE intensity and have a spot shape. These peaks maximum has been analyzed in spectral.

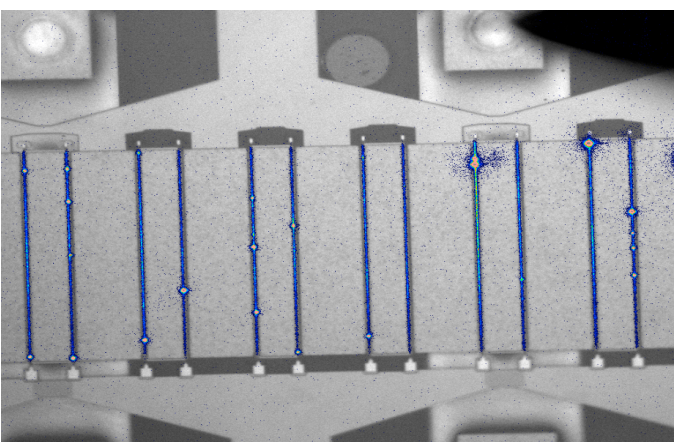

Fig. 6. PE signature of a fresh device in transistor mode: $(\mathrm{Vgs}, \mathrm{Vds})=(-7 \mathrm{~V}, 30 \mathrm{~V})$.

Each spot measured showed no spread of its first order. Thus this is was monochromatic emission. The measured 
wavelength was about $930 \mathrm{~nm}$, corresponding to $1.35 \mathrm{eV}$ level energy.

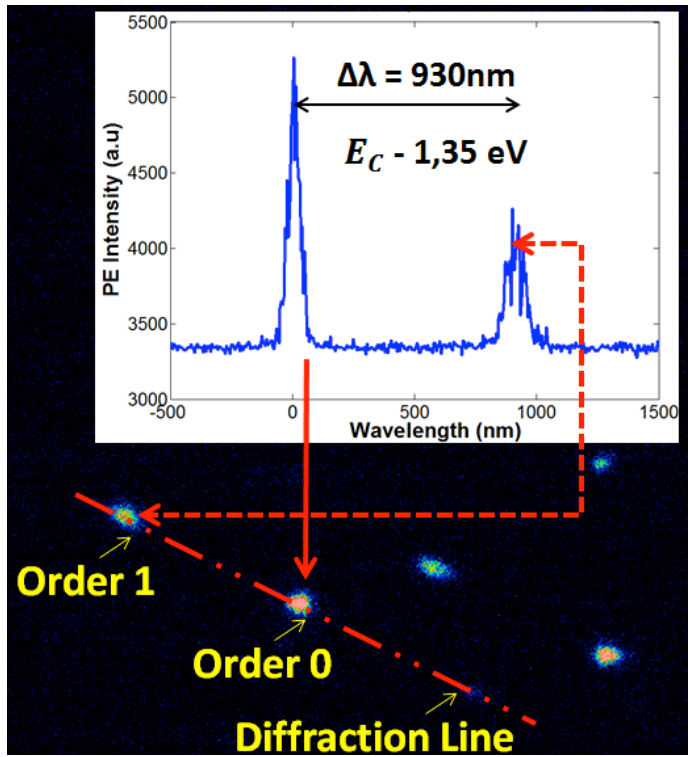

Fig. 7. Photo emission spectrum: Extracting a fresh device spot wavelength in the transistor mode $(\mathrm{VGS}, \mathrm{VDS})=(-7 \mathrm{~V}$, $50 \mathrm{~V})$.

According to literature this deep level energy corresponds either to an interstitial carbon defect that forms along dislocations [14], or an omnipresent defect such as C or possibly dislocations [15].

\subsubsection{Reliability study on a SiC MOSFET: HTGB Stress}

The second device studied in this work is a second generation Cree silicon carbide MOSFETs, having a DMOSFET structure (Fig. 8), available in the market with the voltage and current of $1200 \mathrm{~V}$ and 19 A respectively.

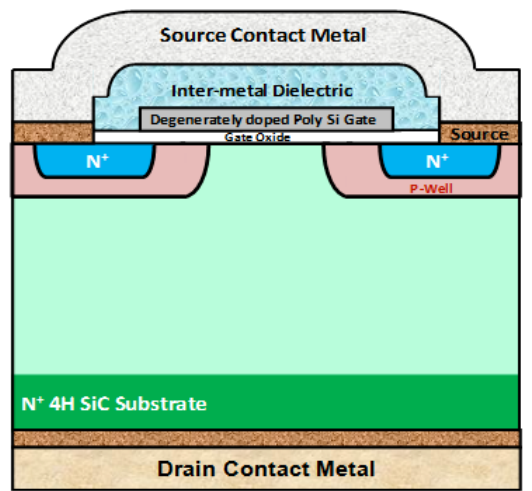

Fig. 8. $2^{\text {sd }}$ Generation SiC Cree MOSFET: D-MOSFET Structure [16]

A device has been aged by high temperature gate bias (HTGB) test with $(\mathrm{Vds} ; \mathrm{Vgs})=(960 \mathrm{~V} ;-5 \mathrm{~V})$ for 26 days. It is a step stress temperature increasing from $120^{\circ} \mathrm{C}$ to $190^{\circ} \mathrm{C}$. Following this aging test, there was an increase of Rds (on), drain leakage current (Idss) and threshold voltage (Vth).
A study by spectral PEM is performed on the aged and the fresh devices (Fig 9). The spectrum of the two devices shown in Fig. 10, presents a decrease in the intensity of PE for the aged component, and a slight shift in the decreasing wavelengths.

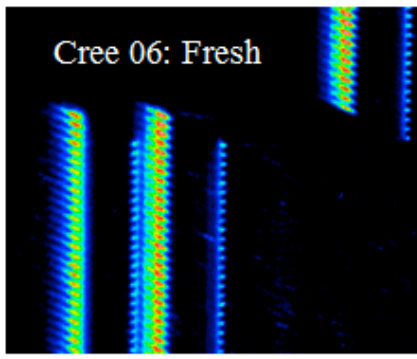

4181

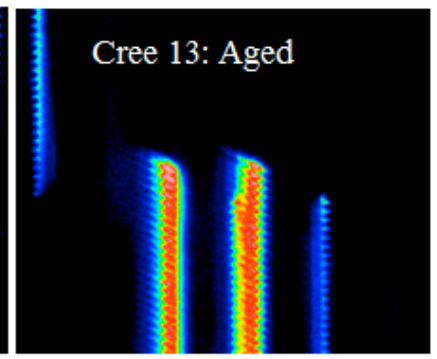

14681
Fig. 9 Spectral PEM analysis of the fresh and the aged devices

It is believed that following the aging test, the surface between the gate oxide and the semi-conductor is damaged. This has degraded the gate command, which explain the decrease of the thershold voltage and the spectrum shift towards the decreasing wavelenghts. In order to prove this hypothesis further analysis in focused ion beam and scanning electron microscopy are planned.

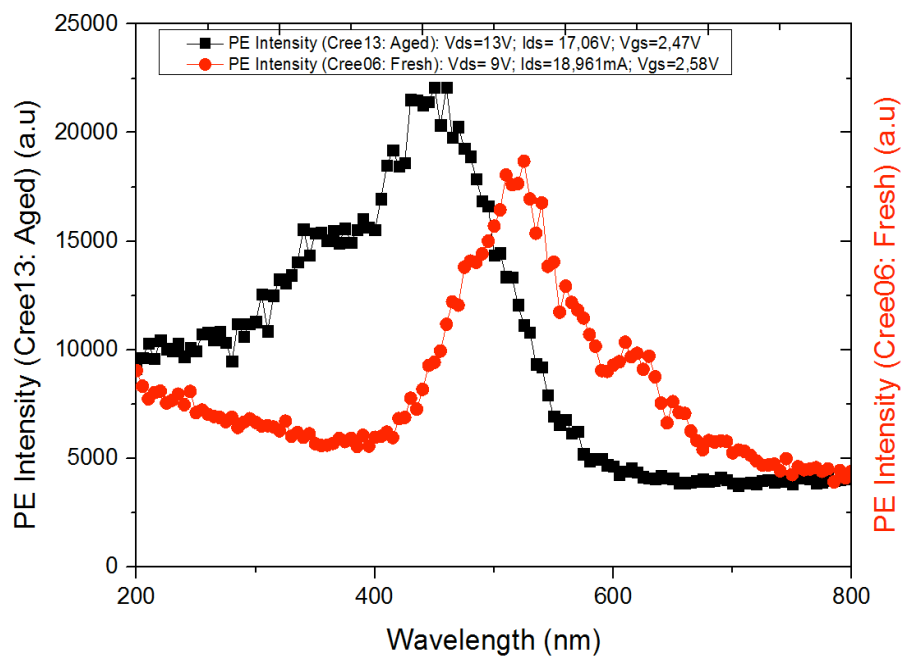

Fig. 10. PE spectrum of the fresh and the HTGB aged devices

\subsubsection{Robustness Study on a SiC MOSFET: ESD Stress}

In this paper, we are interested not only by studying the reliability of this SiC MOSFET devices with the HTGB test, but also in evaluating there robustness to ESD stress, using the spectral PEM technique.

We studied the spectral PE signature of both the fresh and the ESD degraded devices. The PE spectrums obtained are in the frequency range of the visible and near infrared (Fig. 11). A spectrum shift in the increasing wavelengths was noticed, in addition to a decrease in the intensity of light emission between the fresh and the degraded devices. 


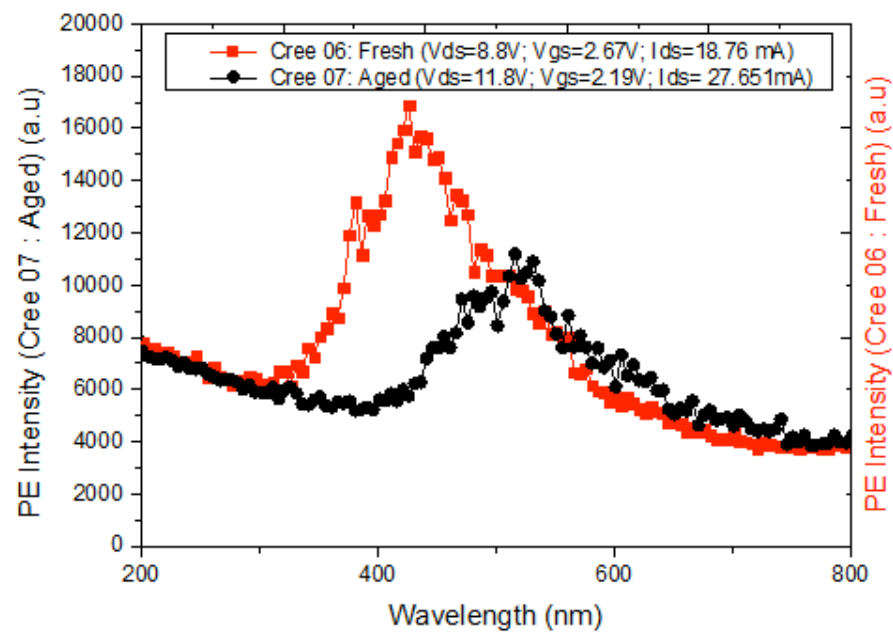

Fig. 11. PE spectrum of the fresh and the ESD aged devices

Following the ESD stress, diffusion at this junction is suspected, which has generated an increase in leakage current, and has caused a spectral gap between the fresh and the degraded device. To confirm this hypothesis Nano-SIMS analyses will be performed.

\section{Conclusions}

In this paper, photon emission microscopy and spectroscopy for microelectronic failure analysis are presented together with the instrumentation developments that allow these techniques to be effectively deployed for microelectronic fault localizing and defect characterization.

We introduced in this paper a new spectral photon emission system. We demonstrate its application on a variety of high power mechatronic devices. The failure mechanisms related to the results spectrums are discussed and further analysis are in perspectives.

\section{Acknowledgements}

This work is funded by the French MFP FIRST project.

\section{References}

[1] S.M. Kudva et al. , The SEMATECH Failure Analysis Roadmap, Proc Int Symp Testing \& Failure Analysis (ISTFA 1995), 6-10 Nov 95, Santa Clara, California, USA, pg 1-5, 1995

[2] I. De Wolf and M.S. Rasras. Spectroscopic photon emission microscopy: a unique tool for failure analysis of microelectronics devices. Microelectronics Reliability, 41(8):1161-1169, August 2001.

[3] J.M. Tao et al., Analysis And Quantification Of Device Spectral Signatures Observed Using A Spectroscopic Photon Emission Microscope, Proceedings of 6th IPFA '97, Singapore 1997.

[4] J. C. H. Phang, D. S. H. Chan, et al. A review of near infrared photon emission microscopy and spectroscopy. In Physical and Failure Analysis of Integrated Circuits, 2005. IPFA 2005. Proceedings of the $12^{\text {th }}$ International Symposium on the, pages 275-281. IEEE, June 2005.
[5] J. Tao, P. Fang, and J. Wang. Backside IR photon emission microscopy (IR-PEM) observation in failure analysis of the packaged devices. In Electronic Packaging Technology, 2007. ICEPT 2007. 8th International Conference on, pages 1-4. IEEE, August 2007.

[6] J. Kolzer, C. Boit, A. Dallmann, G. Deboy, J. Otto, D. Weinmann, "Quantitative Emission Microscopy", J Appl Phys, Vol 70, No 11, pg 23-41,1992.

[7] Y. Y. Liu, J. M. Tao, D. S. H. Chan, J. C. H. Phang, and W. K. Chim. A new spectroscopic photon emission microscope system for semiconductor device analysis. In Physical and Failure Analysis of Integrated Circuits, 1995., Proceedings of the 1995 5th International Symposium on the, pages 60-65. IEEE, November 1995.

[8] D. S. H. Chan, J. C. H. Phang, W. K. Chim, et al. Design and performance of a new spectroscopic photon emission microscope system for the physical analysis of semiconductor devices. Review of Scientific Instruments, 67(7):2576-2583, July 1996.

[9] K.. De Kort, P. Damink ,H. Boots, Spectrum Emitted by Hot Electrons in p-i-n Cold Cathodes, Physical B, Vol 48, No 16,pg 11912-11920, 1993.

[10] K. De Kort and P. Damink, The spectroscopic signature of light emitted by integrated circuits, Proceeding of the European Symposium on Reliability of Electron Devices, Failure Physics and Analysis (ESREF'90), p. 45, 1990.

[11] M.S Rasras, I. De Wolf, G. Groeseneken, and H. E. Maes. Spectroscopic identication of light emitted from defects in silicon devices. Journal of Applied Physics, 89(1):249-258, January 2001.

[12] S.C. Tan, K. Toh, J. Phang, D. Chan, et al. . A NearInfrared, continuous wavelength, In-Lens spectroscopic photon emission microscope system. In Physical and Failure Analysis of Integrated Circuits, 2007. IPFA 2007. 14th International Symposium on the. IEEE, July 2007.

[13] P. Scholz, A. Glowacki, U. Kerst, C. Boitet al. , Single image spectral electroluminescence (photon emission) of GaN HEMTs. In Reliability Physics Symposium (IRPS), 2013 IEEE International, pages CD.3.1-CD.3.7. IEEE, April 2013.

[14] M. Armstrong, Investigation of deep level defects in GaN:C,GaN:Mg AND Pseudomorphic AlGaN/GaN Films; Thesis dissertation University of Ohio, 2006.

[15] A. Armstrong, A. R. Arehart, Impact of carbon on trap states in n-type GaN grown by metalorganic chemical vapor deposition, App. Phy Vol 84, Num 3. 2004.

[16] J. Casady, J. Palmour, High Megawatt Direct-Drive Motors and Front-End Power Electronics Workshop Sept 2014, Panel: WBG Devices Cost and Development Roadmap:

http://www.nist.gov/pml/high megawatt/sept2014 pres entations.cfm 